\title{
Effect of consuming a grape seed supplement with abundant phenolic compounds on the oxidative status of healthy human volunteers
}

\author{
Felix Grases ${ }^{1,2^{*}}$, Rafel M. Prieto ${ }^{1,2}$, Rafel A. Fernández-Cabot ${ }^{1}$, Antonia Costa-Bauzá ${ }^{1,2}$, Ana M. Sánchez ${ }^{3}$
} and Marin Prodanov ${ }^{3}$

\begin{abstract}
Background: Diverse enzymatic and non-enzymatic antioxidants provide protection against reactive oxygen species in humans and other organisms. The nonenzymatic antioxidants include low molecular mass molecules such as plant-derived phenols.

Aim of study: This study identified the major phenolic compounds of a grape seed extract by HPLC and analyzed the effect of consumption of biscuits enriched with this extract on the urinary oxidative status of healthy subjects by measurement of urine redox potential.

Methods: The major phenolic compounds were characterized in a red grape seed extract separated by HPLC with detection by a photodiode array (PDA), fluorescence (FL) and quadrupole mass spectrometer (MS). A nutritional study in a healthy volunteers group was done. Each volunteer ate eight traditional biscuits with no red grape seed extract supplementation. The second day each volunteer ate eight traditional biscuits supplemented with $0.6 \%$ (wt/wt) of grape seed extract. An overnight urine sample was obtained for each treatment. The redox potential was measured at $25^{\circ} \mathrm{C}$ using a potentiometer in each urine sample.
\end{abstract}

Results: Epicatechin, catechin, procyanidin dimers B1 to B4, and the procyanidin trimer C2 were the major phenolic components in the extract. Epicatechin gallate and procyanidin dimers B1-3-G and B2-3'-G were the major galloylated flavan-3-ols. The forty-six healthy volunteers each shown a reduction of the urine redox potential after the treatment by traditional biscuits supplemented with the grape seed extract.

Conclusions: This simple dietary intervention significantly reduced (33\%) the urine redox potential, reflecting an overall increase in antioxidant status. Incorporation of plant-derived phenols in the diet may increase anti-oxidative status.

\section{Background}

Reactive oxygen species (ROS) and free radicals can react with membrane lipids, nucleic acids, and proteins, leading to cellular damage. Humans and other organisms have diverse antioxidant systems that protect against

\footnotetext{
* Correspondence: fgrases@uib.es

'Laboratory of Renal Lithiasis Research, University Institute of Health Sciences Research (IUNICS), University of Balearic Islands, 07122 Palma of Mallorca, Spain

${ }^{2}$ CIBER Fisiopatología de la Obesidad y Nutrición (CB06/03), Instituto de Salud Carlos III, 07122 Palma of Mallorca, Spain

Full list of author information is available at the end of the article
}

ROS. Enzymatic antioxidants include superoxide dismutase, glutathione peroxidase, glutathione transferase, and catalase [1]; nonenzymatic antioxidants include low molecular mass molecules, such as ascorbic acid, vitamin E, uric acid, N-acetylcysteine, carotenoids, phenols, and phytates [2]. When ROS are generated in excess or in amounts that overwhelm antioxidant defense mechanisms, oxidative stress and cell damage may occur. Oxidative stress is associated with the pathogenesis of numerous human diseases, including neurodegenerative diseases [3], psychiatric conditions [4], rheumatoid 
arthritis [5] cancer [6], and renal lithiasis [7]. Thus, there is great emphasis on antioxidant supplement therapy that targets ROS [8].

Grape (Vitis vinfera L.) seed extracts have high levels of numerous anti-oxidants and are considered among the most powerful plant-derived antioxidant foods. Their antioxidant activity is mainly attributed to flavonoids and 3 different flavan-3-ols (flavanols): catechin, epicatechin, and epicatechin gallate and its polymers. Flavan-3-ols can donate electrons or protons to ROS and act as scavengers $[9,10]$. Some authors [11] claimed that the antioxidant activity of these compounds is due to the presence of hydroxyl groups at positions 3, 5, 4', and $5^{\prime}$ (and position 7 to a lesser extent) of the benzopyran structure, and those of the gallic acid moiety in the case of the galloylated forms (Fig. 1). Plumb et al. [12] proposed that antioxidant activity increased from monomers to trimers, and then decreased from trimers to tetramers. Grape seed procyanidins are particularly interesting because of the vast diversity of their polymeric and galloylated forms, which are based on several basic elemental units [13].

The main problem in studying individual grape seed procyanidins is their separation and quantification. Normalphase HPLC can separate flavan-3-ols by molecular mass, but is limited to qualitative or semi-quantitative analysis. Reversed-phase HPLC is mostly used for fine separation of flavan-3-ols, but only allows separation and quantification of monomers, dimers, some nongalloylated trimers, and occasionally tetramers and monogalloylated trimers. In most cases, prior purification of the sample is necessary [14, 15]. Increasing the chromatographic resolution by use of two dimensional chromatography allows separation of heptamer nongalloylated, hexamer monogalloylated, and pentamer digalloylated procyanidins [16], but only provides qualitative results. Even with advances in the development of microbore columns and ultra-high pressure HPLC (UPLC) [17], the main HPLC technique currently used for measurement of flavan-3-ols is based on a $\mathrm{C}_{18}$ bonded stationary phase with $5 \mu \mathrm{m}$ particle size, and this only provides partial quantitative estimates of some individual flavan-3ols.

Urine antioxidant capacity is usually assessed by complex procedures, including assays of total phenolics (Folin-Ciocalteu assay), ferric-reducing antioxidant power (FRAP), oxygen radical absorbance capacity (ORAC) [18], or by determination of the concentrations of individual antioxidant compounds such as malondialdehyde, ascorbic acid, or uric acid. Measurement of urinary redox potential is a simpler method for assessment of urine antioxidant capacity [19].

In the present study, we determined the major phenolic compounds of exGrape ${ }^{\bullet}$ grape seed extract by an improved HPLC method and analyzed the effect of consumption of this product on urine oxidative status by measurement of urinary redox potential in healthy volunteers.

\section{Methods}

\section{Extract characterization, reagents, and apparatuses}

The reference substances, $(+)$-catechin (purity $>99 \%$ ), (-)-epicatechin (purity $>99 \%),(-)$-epicatechin-3-gallate (purity $>97.5 \%$ ), procyanidin dimer $\mathrm{B}_{1}$ (purity $>80 \%$ ), and procyanidin dimer $B_{2}$ (purity $>90 \%$ ), were from Extrasynthèse (Genay, France). Gallic acid (99 \% purity) was from Sigma-Aldrich (St. Louis, Missouri, USA). Procyanidin dimers $B_{3}-B_{7}$ were purified from natural extracts by high-speed counter current chromatography (HSCCC) according to Köhler et al. [20] and Esatbeyoglu et al. [21], and were characterized by nuclear magnetic resonance (NMR). Purified procyanidin extract from cocoa (Breko $\mathrm{GmbH}$, Bremen, Germany) was used as a complex

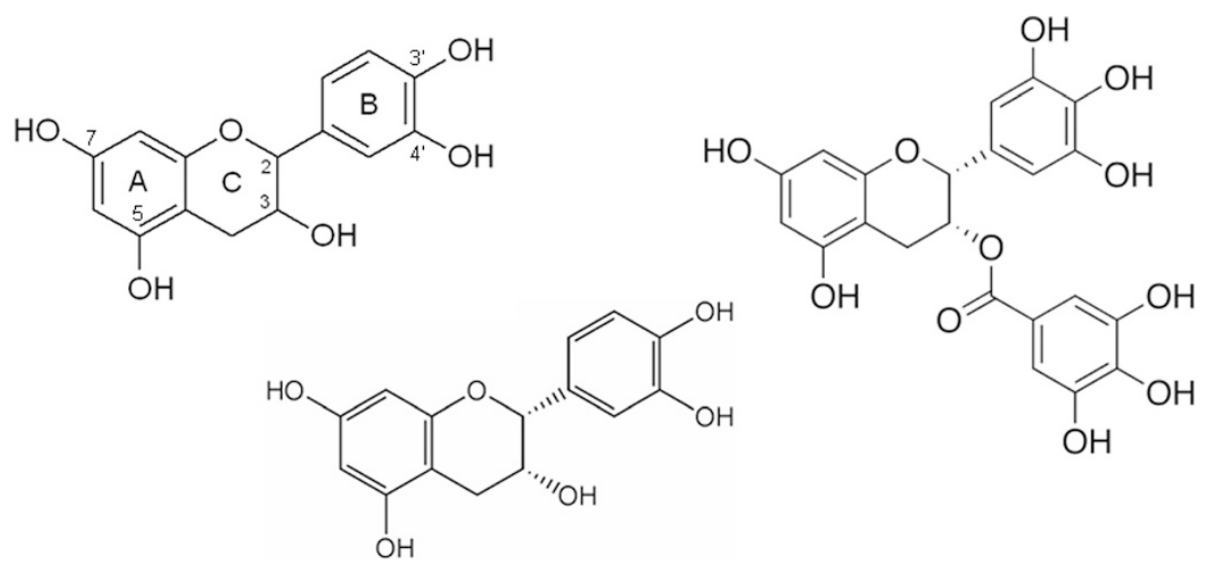

(+)-Catechin

(-)-Epicatechin

(-)-Epigallocatechin

Fig. 1 Chemical structure of the elemental flavan-3-ol units of grape seed extracts 
reference sample for the identification of procyanidin trimer $\mathrm{C}_{1}$ [EC-(4 $\left.\alpha-8\right)$-EC- $(4 \alpha-8)$-EC], tetramer [EC- $(4 \alpha-8)$ EC-( $4 \alpha-8)-E C-(4 \alpha-8)-E C]$, pentamer [EC- $(4 \alpha-8)$-EC-(4a-8)EC- $(4 \alpha-8)-E C-(4 \alpha-8)-E C]$, and hexamer [EC- $(4 \alpha-8)-E C-$ $(4 \alpha-8)-\mathrm{EC}-(4 \alpha-8)-\mathrm{EC}-(4 \alpha-8)-\mathrm{EC}-(4 \alpha-8)-\mathrm{EC}]$.

Grape seed extract constituents were assessed by the reversed phase (RP) HPLC method of Prodanov et al. [15], with adaptation for a $3 \mu \mathrm{m}$ particle size stationary phase to increase the separation efficiency [22]. Two HPLC apparatuses were used: (i) HPLC/PAD/FL, a Varian 920-LC Galaxie (Varian Instruments, Walnut Creek, California, USA) that was coupled to a photodiode array (PDA) and fluorescence (FL) detectors in series and (ii) an Agilent series $1100 \mathrm{HPLC} / \mathrm{PAD} / \mathrm{MS}$ (Palo Alto, California, USA) that was coupled to a quadrupole mass spectrometer (MS) (Hewlett-Packard series 1100 MSD) with an electrospray interface (ESI). Separation was performed on an ACE-3-C18-AR $(200 \mathrm{~mm} \times 4.6 \mathrm{~mm}, 3 \mu \mathrm{m}$ particle size) column from Advanced Chromatography Technologies (Aberdeen, UK) and a guard-column $(20 \times 4.6 \mathrm{~mm})$ of the same material. The mobile phase was a linear gradient of solvent A (water:acetic acid, 98:2) to solvent B (acetonitrile:acetic acid, 98:2) as follows: from 0 to $20 \%$ of $\mathrm{B}$ in $80 \mathrm{~min}$, from 20 to $28 \%$ of B in $35 \mathrm{~min}$, from 28 to $100 \%$ of B in $5 \mathrm{~min}$, isocratically $100 \%$ of B for $10 \mathrm{~min}$, from 100 to $0 \%$ of B in $5 \mathrm{~min}$, and equilibration of the column for the next analysis for $10 \mathrm{~min}$. The total run time was $140 \mathrm{~min}$. The flow rate of the mobile phase was $0.6 \mathrm{~mL} /$ min. Chromatograms were acquired by measurement of absorption at $280 \mathrm{~nm}$ (by the PDA) and fluorescence at $316 \mathrm{~nm}$ (273 nm excitation). For ESI, the drying gas was $\mathrm{N}_{2}\left(10 \mathrm{~L} / \mathrm{min}\right.$ at $\left.340{ }^{\circ} \mathrm{C}\right)$, the nebulizing pressure was $40 \mathrm{psi}$, and the capillary tension was $4000 \mathrm{~V}$. Mass spectra were obtained by scanning negative ions from $\mathrm{m} / \mathrm{z}$ less than 200 at $100 \mathrm{~V}, m / z \quad 200-1000$ at $200 \mathrm{~V}$, and $m / z$ $1000-2500$ at $250 \mathrm{~V}$. Mass spectra were recorded from $\mathrm{m} /$ $z 100$ to 2500 .

Gallic acid, (+)-catechin, (-)-epicatechin, (-)-epicatechin3-O-gallate, and procyanidin dimers $\mathrm{B}_{1}$ and $\mathrm{B}_{2}$ were identified by co-elution and comparison with the masses and UV spectra and retention times of commercial references. Procyanidin dimers $B_{3}, B_{4}, B_{5}$, and $B_{7}$ were identified by comparison of the UV absorbance spectra, fluorescence following excitation at $273 \mathrm{~nm}$, molecular ions, and retention times of reference substances isolated by HSCCC from natural extracts $[20,21]$ and characterized by NMR. Pro-

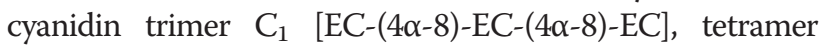
[EC-( $4 \alpha-8)-E C-(4 \alpha-8)-E C-(4 \alpha-8)-E C]$, pentamer [EC-( $4 \alpha-$ 8)-EC-(4 $\alpha-8)-\mathrm{EC}-(4 \alpha-8)-\mathrm{EC}-(4 \alpha-8)-\mathrm{EC}]$, and hexamer [EC$(4 \alpha-8)$-EC-( $4 \alpha-8)$-EC-( $4 \alpha-8)$-EC-(4 $\alpha-8)$-EC-( $4 \alpha-8)$-EC] were identified by comparison of the UV absorbance spectra, fluorescence following excitation at $273 \mathrm{~nm}$, molecular ions, and retention times of the corresponding complex reference sample from a purified procyanidin extract from cocoa [23]. For the procyanidin pentamer [EC-(4 $\alpha-8)$-EC(4 $\alpha-8)$-EC- $(4 \alpha-8)$-EC- $(4 \alpha-8)$-EC], both the single- and the double-charged ions were present, but for the procyanidin hexamer [EC-(4 $\alpha-8)$-EC-( $4 \alpha-8)$-EC-( $4 \alpha-8)$-EC- $(4 \alpha-8)$-EC$(4 \alpha-8)-\mathrm{EC}]$, only the double-charged ion was present in the mass spectra. Procyanidin trimers, $\mathrm{C}_{2}[\mathrm{C}-(4 \alpha-8)-\mathrm{C}-(4 \alpha-8)-$ $\mathrm{C}]$ and $[\mathrm{EC}-(4 \alpha-8)-\mathrm{EC}-(4 \alpha-8)-\mathrm{C}]$, were identified based on mass, UV absorbance spectra, fluorescence following excitation at $273 \mathrm{~nm}$, retention time, and order of elution [15]. The galloylated procyanidin dimers $B_{1}-3-G$ and $B_{2}-3{ }^{\prime}-G$ were identified by mass, UV absorbance spectra, retention times, and order of elution [15].

Gallic acid, (+)-catechin, (-)-epicatechin and (-)-epicatechin-3-gallate were quantified by calibration curves of standards. All procyanidin nongalloylated dimers to hexamers were referred to the calibration curve of the procyanidin dimer $B_{1}$ from the fluorescence chromatograms. The quantities of galloylated procyanidin dimers $B_{1}-3-G$ and $B_{2}-3$ '-G were calculated based on a calibration curve for the (-)-epicatechin-3-gallate from the $280 \mathrm{~nm}$ absorbance chromatograms.

\section{Human studies}

Forty-six non-smokers healthy volunteers (20 males and 26 females, mean age: 34 years, age range: 17 to 60 years) were invited to participate. Individuals who reported consuming antioxidant supplements or omega-3 polyunsaturated fatty acids and those with addiction to alcohol or drugs were excluded. None of the participants received any pharmacological treatment during urine collection. Fruits and vitamin supplements were discontinued $24 \mathrm{~h}$ prior to collection of the first urine sample. All subjects were instructed to maintain their usual diets and to not participate in sports activities during the two day study period. The subjects consumed the biscuits with the $100 \%$ of compliance. All subjects provided written informed consent and the study protocol (IB 2030/13 PI) was approved by the Ethics Investigation Committee of the Balearic Islands (Spain).

The first day, each volunteer ate eight traditional biscuits (Quely from Quely S.A., Mallorca Islands, Spain) during the day (4 in breakfast and 4 in dinner) with no plant-derived phenol supplementation. The biscuits, of $9 \mathrm{~g}$ of weight, are made with wheat, sunflower oil, yeast, olive oil and salt. An overnight urine sample, starting prior to sleep and ending with the first morning urine while still in a fasting state, was obtained the next day.

The second day each volunteer ate eight traditional biscuits supplemented with $0.6 \%$ (wt/wt) of red grape (Vitis vinfera $L$.) seed extract (Quely Cor from Quely S.A.,) during the day, corresponding to about $250 \mathrm{mg}$ of phenols, the phenols content of the biscuits was measured by Folin's method and expressed as gallic acid. This represent a total 
polyphenol intake about $2 \mathrm{~g} / \mathrm{d}$. The red grape seed extract (exGrape ${ }^{\circ}$ ) was purchased from La Gardonnenque-Groupe Grap'Sud $^{\circ}$ (Cruviers-Lascours, France). The second urine sample was collected on the third morning as described above.

At $2 \mathrm{~h}$ after collection of each non filtered urine sample, the redox potential was measured at $25{ }^{\circ} \mathrm{C}$ using a Crison potentiometer (Crison Instruments S.A., Barcelona, Spain), with a platinum electrode as the working electrode and a saturated calomel electrode as the reference electrode. Results are shown as means \pm standard errors and compared using Student's paired $t$-test.

\section{Results}

Table 1 shows the chemical composition, the mass spectral data and the content of of the grape seed extract exGrape ${ }^{\circ}$ used in this study. Figure 2 illustrates a typical ultraviolet absorption chromatogram $(280 \mathrm{~nm})$ of the studied extract, with indications of the main identified compounds. The results indicates that this extract mainly consists of flavan-3-ol monomers and oligomers (17.46/
$100 \mathrm{mg}$ ), and very small amounts of gallic acid and some other unidentified compounds. Epicatechin, catechin, procyanidin dimers $\mathrm{B}_{1}$ to $\mathrm{B}_{4}$, and the procyanidin trimer $\mathrm{C}_{2}$ were the major components, and these had concentrations of $0.65-1.70 / 100 \mathrm{~g}$. Lower amounts of other nongalloylated procyanidin trimers and the procyanidin tetramer [EC-EC-EC-EC] were also present. The improved HPLC method detected the procyanidin pentamer [EC-EC-ECEC-EC] and hexamer [EC-EC-EC-EC-EC-EC], but their amounts were lower than the limit needed for quantification by the fluorescence detector. The improved HPLC method also detected the procyanidin pentamer [EC-ECEC-EC-EC] and hexamer [EC-EC-EC-EC-EC-EC], but their amounts were also lower than the limit needed for quantification. The total amount of the nongalloylated procyanidin monomers and oligomers was 9.11/100 mg and the amount of the galloylated forms (represented only by the epicatechin gallate and the common peak of the monogalloylated dimers $B_{1}-3-G$ and $\left.B_{2}-3^{\prime}-G\right)$, was 0.65 / $100 \mathrm{mg}$.

Table 1 Chemical composition, mass spectral data (negative ionisation mode) and content of the main identified constituents of exGrape ${ }^{\circledast}$ seed extract (quantitaive data are reffered to the extrac as it is)

\begin{tabular}{|c|c|c|c|c|}
\hline $\begin{array}{l}R_{\mathrm{t}} \\
(\min )\end{array}$ & Compound & $\begin{array}{l}\mathrm{M}-\mathrm{H}]^{-} \\
(\mathrm{m} / \mathrm{z})\end{array}$ & $\begin{array}{l}{[\mathrm{M}-2 \mathrm{H}]^{2-}} \\
(\mathrm{m} / \mathrm{z})\end{array}$ & $\begin{array}{l}\text { Content } \\
(\mathrm{mg} / 100 \mathrm{mg})\end{array}$ \\
\hline 8.3 & Gallic acid & 169.0 & & 0.09 \\
\hline 32.2 & $P C_{2}\left(B_{3}\right)[C-C]+P C_{3}\left(C_{2}\right)[C-C-C]$ & $577.0,865.1$ & & 0.65 \\
\hline 34.8 & $P C_{2}\left(B_{1}\right)[E C-C]$ & 577.0 & & 1.19 \\
\hline 36.3 & C & 289.0 & & 1.47 \\
\hline 41.3 & $P C_{3}[E C-E C-C]$ & 865.1 & & 0.30 \\
\hline 47.1 & $\mathrm{PC}_{2}\left(\mathrm{~B}_{4}\right)[\mathrm{C}-\mathrm{EC}]$ & 577.1 & & 0.64 \\
\hline 54.4 & $\mathrm{PC}_{2}\left(\mathrm{~B}_{2}\right)[\mathrm{EC}-\mathrm{EC}]$ & 577.0 & & 1.69 \\
\hline 61.7 & EC & 289.0 & & 1.70 \\
\hline 72.4 & $P C_{2}-G\left(B_{1}-3-G+B_{2}-3^{\prime}-G\right)$ & 729.1 & & 0.51 \\
\hline 74.8 & $\mathrm{PC}_{2}\left(\mathrm{~B}_{7}\right)[\mathrm{EC}-\mathrm{C}]$ & 577.1 & & 0.23 \\
\hline 76.0 & $\mathrm{PC}_{3}\left(\mathrm{C}_{1}\right)[\mathrm{EC}-\mathrm{EC}-\mathrm{EC}]$ & 865.1 & & 0.83 \\
\hline 81.6 & $\mathrm{PC}_{4}[\mathrm{EC}-\mathrm{EC}-\mathrm{EC}-\mathrm{EC}]$ & 1153.1 & & 0.28 \\
\hline 83.2 & $\mathrm{PC}_{5}[\mathrm{EC}-\mathrm{EC}-\mathrm{EC}-\mathrm{EC}-\mathrm{EC}]$ & 1441.1 & 720.2 & $0.11^{a}$ \\
\hline 85.0 & $\mathrm{PC}_{6}[\mathrm{EC}-\mathrm{EC}-\mathrm{EC}-\mathrm{EC}-\mathrm{EC}-\mathrm{EC}]$ & - & 864.2 & $0.02^{a}$ \\
\hline 92.5 & EC-G & 441.1 & & 0.14 \\
\hline 111.2 & $\mathrm{PC}_{2}\left(\mathrm{~B}_{5}\right)[\mathrm{EC}-\mathrm{EC}]$ & 577.2 & & 0.22 \\
\hline & \multicolumn{3}{|c|}{$\sum$ nongalloylated flavan-3-ol monomers } & 3.17 \\
\hline & \multicolumn{3}{|l|}{$\sum$ nongalloylated flavan-3-ol dimers } & 4.53 \\
\hline & \multicolumn{3}{|c|}{$\begin{array}{l}\sum \text { nongalloylated flavan-3-ol monomers and } \\
\text { oligomers }\end{array}$} & 9.11 \\
\hline & \multicolumn{3}{|c|}{$\sum$ galloylated flavan-3-ol monomer and oligomers } & 0.65 \\
\hline & \multicolumn{3}{|l|}{$\sum$ flavan-3-ols } & 17.46 \\
\hline
\end{tabular}

$R_{t}$ retention time, $C(+)$-catechin, $E C(-)$-epicatechin, $P C_{x}$ procyanidin oligomers (subscript $x$ - number of elemental units $\left(x=2\right.$ (dimer), 3 (trimer), etc.), $P C_{x} G$ galloylated procyanidin, $G$ galloyl unit

a Values below the limit of quantification 


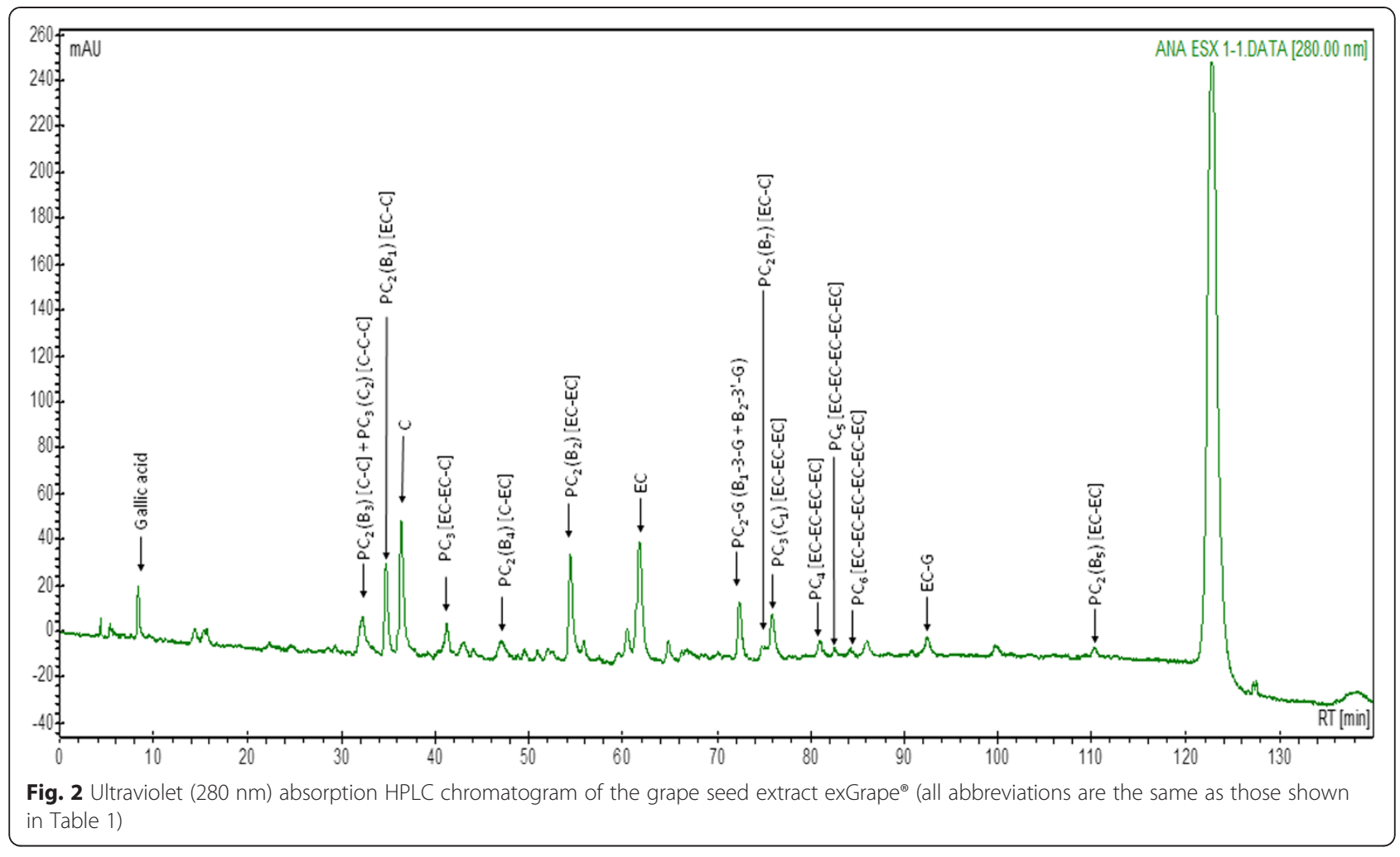

Figure 3 shows the redox potential of the urine from 46 healthy volunteers at $12 \mathrm{~h}$ after consumption of eight traditional biscuits and at $12 \mathrm{~h}$ after consumption of eight traditional biscuits with grape seed extract, the redox potential range observed before consuming the

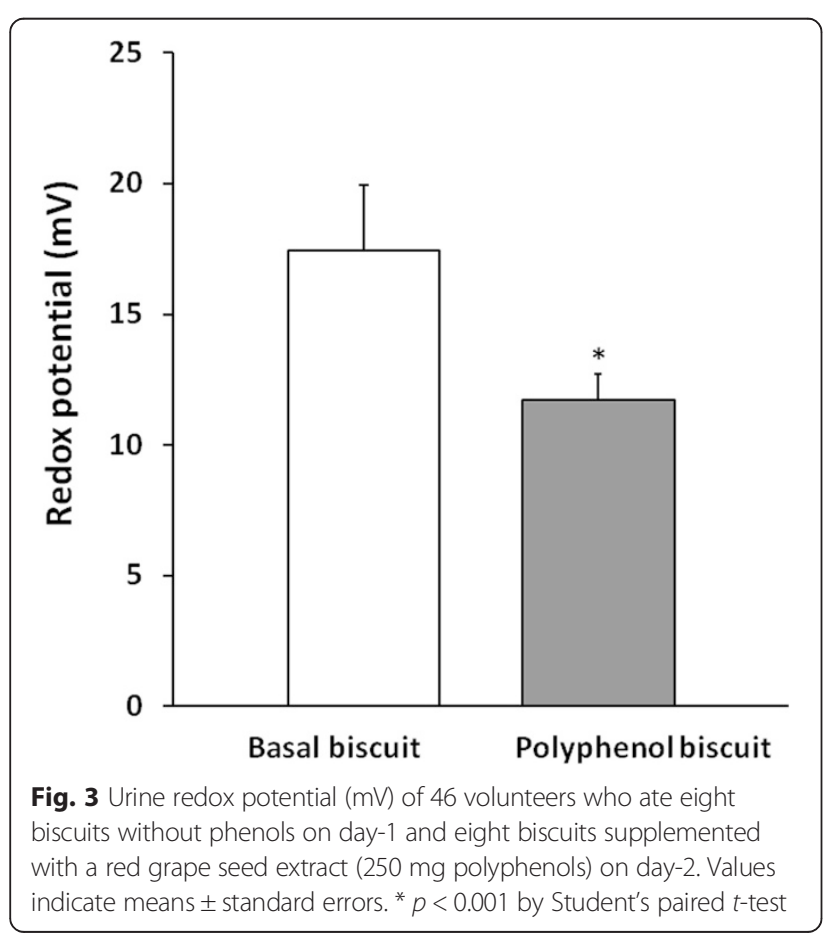

phenolic compound rich biscuits was $76 \mathrm{mV}$ and after consuming the phenolic compound rich biscuits of $114 \mathrm{mV}$. The urinary redox potential was $33 \%$ lower after consumption of the grape seed extract, indicating that consumption of this polyphenol-rich supplement significantly increased the antioxidant capacity of urine.

\section{Discussion}

Table 1 shows that the exGrape ${ }^{\circ}$ seed extract has a composition typical of grape seed extracts, in that the monomers catechin and epicatechin are most abundant, followed by the nongalloylated procyanidin dimers and trimers with $\mathrm{C}_{4}-\mathrm{C}_{8}$ interflavan bonds $[14,15]$. Epicatechin and epicatechin-based oligomers (procyanidin dimer $B_{2}$ and trimer $C_{1}$ ) are the most abundant among the other catechin-containing derivatives. The natural fluorescence of the nongalloylated flavan-3-ols was greater, so the procyanidin tetramer, [EC-EC-EC-EC], was quantified whereas the other two procyanidin oligomers, [EC-EC-EC-EC-EC] and [EC-EC-EC-EC-EC-EC], were simply separated and detected. To the best of our knowledge this is the first time that pentamer and hexamer procyanidins were separated and detected in a single RP HPLC run with direct injection of an unpurified grape seed extract. The content of flavan-3-ol monomers and oligomers was within the normal limits for this type of extract, even though the level was almost 2-times lower than reported by Shafiee et al. [24] for the same 
extract 10 years ago. However, the difference with regard to the total content of nongalloylated flavan-3-ol monomers and dimmers was minimal, suggesting that the differences are most probably due to use of different analytical methods. In particular, Shafiee et al. [24] used the vanillin method for assessment of oligomer procyanidins, and this method is based on vanillin condensation of all procyanidins in the sample, including high molecular mass procyanidin polymers. In contrast, we only considered the major and well-resolved procyanidin peaks, and these do not account for all nongalloylated procyanidins.

Grape seed extracts differ from other flavan-3-ol-rich extracts because of their high content of galloylated compounds. In some red varieties of grapes, these can account for up to $18 \%$ of the total procyanidin content [14]. Some authors consider these types of procyanidins as stronger antioxidants than the corresponding nongalloylated analogues $[11,12]$, and this has led to increased interest in the galloylated forms. Nevertheless, aside from the present study, there is little quantitative data on the levels of galloylated and nongalloylated compounds in grape seed extracts. Thus, even with our improved chromatographic resolution achieved by use of the $3 \mu \mathrm{m}$ particle size stationary phase, we were only able to separate and unambiguously quantify the epicatechin gallate and a peak containing two monogalloylated dimers, the $B_{1}-3-G$ and $B_{2}-3^{\prime}-G$. Perhaps, the most noteworthy difference we found is that the epicatechin gallate content was 10-fold lower than reported by Shafiee et al. [24] for the same extract. Nevertheless, we should note that we detected many mono- and digalloylated procyanidin oligomers in the extract, but their separation was poor and their amounts were close to or below to limits of detection.

Plant-derived phenols are important dietary antioxidants, and the main dietary sources are fruits and fruit juices. Tea, red wine, vegetables, legumes, cereals, and chocolate also contribute to total polyphenol intake, which may be as high as $1 \mathrm{~g} /$ day [25]. Grape seed extract is a concentrated source of polyphenols [26]. Clinical studies found that consumption of polyphenols can contribute to the prevention of cardiovascular disease [27], some types of cancer [28], osteoporosis [29], and calcium oxalate papillary renal stones [30]. The antioxidant activity of polyphenols prevents oxidative membrane damage through metal chelation and scavenging of ROS [24]. The polyphenol content of urine is an indicator of the intake of polyphenol-rich foods, such as cacao [31]. Moreover, total urinary phenols were significantly increased at $4 \mathrm{~h}$ after the ingestion of red wine that contained $125 \mathrm{mg}$ of polyphenols [gallic equivalent] [32]. The flavonoid concentration in urine, determined by liquid chromatography-mass spectrometry, may reflect the intake of fruits and vegetables, and the total urinary excretion of flavonoids over $24 \mathrm{~h}$ may be a biomarker for fruit and vegetable intake [33].

Urine is the product of blood filtration, and its composition (including that of ions and low molecular mass molecules, such as salts, vitamins, and minerals) mirrors that of blood, despite every subject will have different metabolic rate and therefore different levels of phenolic metabolites could be found in the urine. Thus, a high level of antioxidant tissue destruction may be reflected by decreased urinary excretion of low molecular mass antioxidants. If antioxidants cannot prevent free radical damage or if radical formation is excessive, the oxidant/ antioxidant ratio increases and leads to oxidative stress [34]. The urinary total antioxidant capacity determined is dependent on the levels of non-enzymatic antioxidants, which would be predominantly phenolic metabolites in this case, rather than host derived free radicals, oxidants or electrophiles. One method used to determine the antioxidant capacity of a biological fluid, such as urine, is based on the reduction of ferric to ferrous ions; ferrous ions form complexes with specific dyes and generate a product that can be measured spectrophotometrically [35, 36]. The redox potential of an aqueous solution is a measure of its ability to be oxidized or reduced, and a more negative potential indicates a greater reducing capacity. In contrast, strong oxidizing agents have more positive redox potentials, so a decrease in the urine antioxidant status may reflect an increase in urinary redox potential. Individuals with high oxidative stress will therefore excrete urine with low levels of antioxidants and high redox potential. The correlation of the antioxidant capacity of urine, evaluated using ferric ion and the dye 1, 10-phenantroline (FRAP method) $[35,36]$ with the redox potential using the platinum electrode, was already demonstrated [19]. The correlation was statistically significant by a linear regression equation between antioxidant capacity $\left(\mathrm{mM}\right.$ of $\left.\mathrm{Fe}^{2+}\right)$ or FRAP method versus the redox potential $(\mathrm{mV})(y=-0.073 x+$ 16.941; R:0.522; $\mathrm{p}<0.001$ ) [19]. It demonstrates the suitability of the latter procedure to determine the antioxidant capacity of urine.

The results obtained in this study demonstrate that a single dietary intervention with a grape seed supplement significantly lowered the redox potential of urine, reflecting an overall increase in antioxidant capacity. Other studies have reported similar results after consumption of polyphenol-rich beverages and/or foods [32, 37-39].

\section{Conclusions}

The ExGrape ${ }^{\bullet}$ extract has a composition typical of grape seed extracts, in that the monomers catechin and epicatechin are the most abundant constituents, followed by the nongalloylated procyanidin dimers and trimers with $\mathrm{C}_{4}-\mathrm{C}_{8}$ interflavan bonds. Epicatechin and epicatechin- 
based oligomers (procyanidin dimer $B_{2}$ and trimer $C_{1}$ ) are the most abundant among the other catechincontaining derivatives. Among the nongalloylated flavan3-ols, we quantified the procyanidin tetramer [EC-ECEC-EC] and separated and detected two other procyanidin oligomers, [EC-EC-EC-EC-EC] and [EC-EC-EC-EC$\mathrm{EC}-\mathrm{EC}]$. The phenolic content of urine is an indicator of the intake of plant-derived phenol-rich foods. A single dietary intervention - ingestion of biscuits rich in plantderived phenols - significantly lowered the redox potential of urine, and this reflects an overall increase in antioxidant capacity. The clinical benefits of more sustained dietary interventions with grape seed extract remain to be established.

\section{Competing interests}

The University of Balearic Islands has an agreement to develop biscuits supplemented with red grape seed extract with Quely S.A. (Inca, Spain), whose responsible are FG, RMP and ACB. The other authors declare that they have no competing interests.

\section{Authors' contributions}

FG took part in planning the study design, and drafting and writing of manuscript. RMP took part in planning the study design and drafting and writing of the manuscript. RAFC and ACB were responsible for human studies and took part in planning the study design. AMS and MP contributed to extract characterization, study design and contributed to writing of the manuscript. All authors read and approved the final manuscript.

\section{Acknowledgments}

This work was supported by grant CTQ2010-18271 from the Ministerio de Ciencia e Innovación (Gobierno de España), by FEDER funds (European Union), and by grant 9/2011 from the Conselleria d'Educació, Cultura i Universitat (Govern de les Illes Balears). CIBER Fisiopatología Obesidad y Nutrición (CB06) 03), Instituto de Salud Carlos III, Spain, also provided support. The authors want to thank Quely S.A. (Inca, Spain) for supply the traditional biscuits (Quely) and the biscuits supplemented with red grape seed extract (Quely Cor).

\section{Author details}

'Laboratory of Renal Lithiasis Research, University Institute of Health Sciences Research (IUNICS), University of Balearic Islands, 07122 Palma of Mallorca, Spain. ${ }^{2}$ CIBER Fisiopatología de la Obesidad y Nutrición (CB06/03), Instituto de Salud Carlos III, 07122 Palma of Mallorca, Spain. ${ }^{3}$ Instituto de Investigación en Ciencias de la Alimentación (CIAL) (CEI, CSIC-UAM), Madrid, Spain.

Received: 22 May 2015 Accepted: 7 September 2015

Published online: 09 September 2015

\section{References}

1. Mates JM, Perez-Gomez C, Nunez de Castro I. Antioxidant enzymes and human diseases. Clin Biochem. 1999;32:595-603.

2. Seifried $H E$, Anderson DE, Fisher El, Milner JA. A review of the interaction among dietary antioxidants and reactive oxygen species. J Nutr Biochem. 2007;18:567-79.

3. Barnham KJ, Masters CL, Bush Al. Neurodegenerative diseases and oxidative stress. Nature Rev Drug Discov. 2004;3:205-14.

4. Ozcan ME, Gulec M, Ozerol E, Polat R, Akyol O. Antioxidant enzyme activities and oxidative stress in affective disorders. Int Clin Psychopharmacol. 2004;19:89-95.

5. Datta S, Kundu S, Ghosh P, De S, Ghosh A, Chatterjee M. Correlation of oxidant status with oxidative tissue damage in patients with rheumatoid arthritis. Clin Rheumatol. 2014;33:1557-64.

6. Jezierska-Drutel A, Rosenzweig SA, Neumann CA. Role of oxidative stress and the microenvironment in breast cancer development and progression. Adv Canc Res. 2013;119:107-25.
7. Amengual-Cladera E, Nadal-Casellas A, Gomez-Perez Y, Gomila I, Prieto RM, Proenza AM, et al. Phytotherapy in a rat model of hyperoxaluria: the antioxidant effects of quercetin involve serum paraoxonase 1 activation. Exp Biol Med (Maywood). 2011;236:1133-8.

8. Rodrigo R, Miranda A, Vergara L. Modulation of endogenous antioxidant system by wine polyphenols in human disease. Clin Chim Acta. 2011;412:410-42.

9. Cook NC, Samman S. Flavonoids - chemistry, metabolism, cardioprotective effects, and dietary sources. J Nutr Biochem. 1996;7:66-76.

10. Aron P, Kennedy JA. Flavan-3-ols: Nature, occurrence ans biological activity. Mol Nutr Food Res. 2008;52:79-104.

11. Rice-Evans CA, Miller NJ, Paganga G. Structure-antioxidant activity relationships of flavonoids and phenolic acids. Free Radic Biol Med. 1996;20:933-56.

12. Plumb GW, de Pascual-Teresa S, Santos-Buelga C, Cheynier V, Williamson G. Antioxidant properties of catechins and proanthocyanidins: effect of polymerisation, galloylation, and glycosilation. Free Radic Res. 1998;29:351-8.

13. Waterhouse AL, Ignelzi S, Shirley JR. A comparison of methods for quantifying oligomeric proanthocyanidins from grape seed extracts. Am J Enol Vitic. 2000;51:383-9.

14. Santos-Buelga C, Francia-Aricha EM, Escribano-Bailón MT. Comparative flavan-3-ol composition of seeds from different grape varieties. Food Chem. 1995:53:197-201.

15. Prodanov M, Vacas $V$, Hernández T, Estrella I, Amador B, Winterhalter $\mathrm{P}$. Chemical characterisation of Malvar grape seeds (Vitis vinifera L.) by ultrafiltration and RP-HPLC-PAD-MS. J Food Compost Anal. 2013;31:284-92.

16. Amador-Rojo B. Diseño de bebidas de uso específico para la salud. Spain: PhD thesis, Universidad Autónoma de Madrid; 2012.

17. Montero L, Herrero M, Prodanov M, Ibañez E, Cifuentes A. Characterization of grape seed procyanidins by comprehensive two-dimensional hydrophilic interaction $x$ reversed phase liquid chromatography coupled to diode array detection and tandem mass spectrometry (HILICXRPLC-DAD-MS/MS). Anal Bioanal Chem. 2013:405:4627-38.

18. Goodrich KM, Neilson AP. Simultaneous UPLC-MS/MS analysis of native catechins and procyanidins and their microbial metabolites in intestinal contents and tissues of male Wistar Furth inbred rats. J Chrom B. 2014;958:63-74.

19. Huang D, Ou B, Prior RL. The chemistry behind antioxidant capacity assays. J Agric Food Chem. 2005;53:1841-56.

20. Grases G, Colom MA, Fernandez RA, Costa-Bauzá A, Grases F. Evidence of higher oxidative status in depression and anxiety. Oxid Med Cell Longev. 2014;2014:430216. doi:10.1155/2014/430216.

21. Köhler $N$, Wray $V$, Winterhalter $P$. Preparative isolation of procyanidins from grape seed extracts by high-speed counter-current chromatography. J Chrom A. 2008;1177:114-25.

22. Esatbeyoglu $T$, Wray $V$, Winterhalter $P$. Dimeric procyanidins: screening for B1 to B8 and semisynthetic preparation of B3, B4, B6, and B8 from a polymeric procyanidin fraction of white willow bark (Salix alba). J Agric Food Chem. 2010;58:7820-30.

23. Tomás-Barberán FA, Cienfuegos-Jovellanos E, Marín A, Muguerza B, GilIzquierdo A, Cerdá B, et al. A new process to develop a cocoa powder with higher flavonoid monomer content and enhanced bioavailability in healthy humans. J Agric Food Chem. 2007;55:3926-35.

24. Shafiee M, Carbonneaua MA, Urban N, Descompsa D, Leger CL. Grape and grape seed extract capacities at protecting LDL against oxidation generated by $\mathrm{Cu} 2+$, AAPH or SIN-1 and at decreasing superoxide THP-1 cell production. A comparison to other extracts or compounds. Free Rad Res. 2003;37:573-84.

25. Scalbert A, Williamson G. Dietary intake and bioavailability of polyphenols. J Nut. 2000;130:2073S-85.

26. Ward NC, Croft KD, Puddey IB, Hodgson JM. Supplementation with grape seed polyphenols results in increased urinary excretion of 3hydroxyphenylpropionic acid, an important metabolite of proanthocyanidins in humans. J Agric Food Chem. 2004;52:5545-9.

27. Kris-Etherton PM, Keen CL. Evidence that the antioxidant flavonoids in tea and cocoa are beneficial for cardiovascular health. Curr Opin Lipididol. 2002;13:41-9.

28. Martin MA, Goya L, Ramos S. Potential for preventive effects of cocoa and cocoa polyphenols in cancer. Food Chem Toxicol. 2013;56:336-35. 
29. Shen $\mathrm{CL}$, von Bergen V, Chyu MC, Jenkins MR, Mo H, Chen $\mathrm{CH}$, et al. Fruits and dietary phytochemicals in bone protection. Nut Res. 2012;32:897-910.

30. Grases F, Costa-Bauzá A, Bonarriba CR, Pieras EC, Fernandez RA, Rodriguez A. On the origin of calcium oxalate monohydrate papillary renal stones. Urolithiasis. 2015;43 Suppl 1:33-9.

31. Medina-Remon A, Tresserra-Rimbau A, Arranz S, Estruch R, LamuelaRaventos RM. Polyphenols excreted in urine as biomarkers of total polyphenol intake. Bioanalysis. 2012;4:2705-13.

32. Duthie GG, Pedersen MW, Gardner PT, Morrice PC, Jenkinson AM, McPhail $D B$, et al. The effect of whisky and wine consumption on total phenol content and antioxidant capacity of plasma from healthy volunteers. Eur J Clin Nut. 1998;52:733-6.

33. Krogholm KS, Haraldsdottir J, Knuthsen P, Rasmussen SE. Urinary total flavonoid excretion but not 4-pyridoxic acid or potassium can be used as a biomarker for the intake of fruits and vegetables. J Nut. 2004;134:445-51.

34. McCord JM. Human disease, free radicals, and the oxidant/antioxidant balance. Clin Biochem. 1993:26:351-7.

35. Benzie IF, Strain JJ. The ferric reducing ability of plasma (FRAP) as a measure of "antioxidant power": the FRAP assay. Anal Biochem. 1996;239:70-6.

36. Hayes WA, Mills DS, Neville RF, Kiddie J, Collins LM. Determination of the molar extinction coefficient for the ferric reducing/antioxidant power assay. Anal Biochem. 2011:416:202-5.

37. Cao G, Russell RM, Lischner N, Prior RL. Serum antioxidant capacity is increased by consumption of strawberries, spinach, red wine or vitamin $\mathrm{C}$ in elderly women. J Nut. 1998;128:2383-90.

38. Price RK, Welch RW, Lee-Manion AM, Bradbury I, Strain JJ. Total phenolics and antioxidant potential in plasma and urine of humans after consumption of wheat bran. Cereal Chem. 2008:85:152-7.

39. Roura E, Andres-Lacueva C, Estruch R, Lamuela-Raventos RM. Total polyphenol intake estimated by a modified Folin-Ciocalteu assay of urine. Clin Chem. 2006;52:749-52

\section{Submit your next manuscript to BioMed Central and take full advantage of:}

- Convenient online submission

- Thorough peer review

- No space constraints or color figure charges

- Immediate publication on acceptance

- Inclusion in PubMed, CAS, Scopus and Google Scholar

- Research which is freely available for redistribution 